\title{
CHARACTERISATION OF PECTOBACTERIUM WASABIAE CAUSING BLACKLEG AND SOFT ROT DISEASES IN SOUTH AFRICA
}

Lucy N Moleleki, Edward N Onkendi, Aobakwe Mongae and Gugulethu C Kubheka

\section{Moleleki LN}

Department of Microbiology and Plant Pathology, University of Pretoria, South Africa Forestry Agriculture and Biotechnology Institute

Email: lucy.moleleki@up.ac.za

Telephone: +27 124204662 (Corresponding author)

Fax: +2712420 3266

Onkendi EN

Department of Microbiology and Plant Pathology, University of Pretoria, South Africa Email: eddy.onkendi@fabi.up.ac.za

Mongae A

Department of Microbiology and Plant Pathology, University of Pretoria, South Africa Email: aobakwe.mongae@fabi.ac.za

Kubheka GC

Department of Microbiology and Plant Pathology, University of Pretoria, South Africa Email: s26121702@tuks.co.za 


\begin{abstract}
Pectolytic bacteria were isolated from potato tubers and stems showing tuber soft rot and blackleg symptoms. Approximately half (52\%) of the isolates could grow at both 27 and 37 ${ }^{\circ} \mathrm{C}$ while another half $(48 \%)$ failed to grow at $37{ }^{\circ} \mathrm{C}$. All isolates could be amplified with primers specific to the pectate lyase ( $\mathrm{pel}$ ) gene. Carbon utilisation profiles could not conclusively identify these isolates. PCR amplification using primers specific for Pectobacterium carotovorum subsp. brasiliensis was positive for all isolates that grew at $37^{\circ} \mathrm{C}$. However, the group that did not grow at $37{ }^{\circ} \mathrm{C}$ failed to amplify with $P$. atrosepticum specific primers. To characterise this group of isolates, the intergenic transcribed spacer region (ITS) was amplified and PCR products digested with two restriction enzymes (RsaI and CfoI) to generate ITS-PCR-RFLP profiles. The profiles of these new isolates were compared to those of the type strains of other pectolytic bacteria. Profiles of five of the selected atypical strains generated with the enzyme CfoI appeared to be most similar to those of $P$. wasabiae type strain. Phylogenetic analysis using concatenated partial gene sequences of housekeeping genes $m d h$ and gapA clustered these isolates together with those of $P$. wasabiae reference strains thus confirming their identity. These strains were virulent on potato tubers and stems but did not elicit hypersensitive response on tobacco plants. This is the first report of $P$. wasabiae causing soft rot and blackleg of potatoes in South Africa.
\end{abstract}

Key words: T3SS; soft rot Erwinia; Emerging soft rot Enterobacteriaceae 


\section{Introduction}

Pectobacterium and Dickeya species are members of the family Enterobacteriaceae. Because of their pectolytic nature, they are generally known as soft rot Erwinia or more recently Enterobacteriaceae (SRE) (Charkowski et al., 2012). The SRE have undergone several taxonomic, classification and nomenclature changes and as a result, the former Erwinia carotovora and E. chyrsanthemi species were subdivided into the genus Pectobacterium and Dickeya respectively based on biochemical, molecular and host range differences (Hauben et al., 1998; Gardan et al., 2003; Samson et al., 2005).

The genus Pectobacterium has been divided into four species and these are Pectobacterium atrosepticum $(P a), P$. wasabiae $(P w), P$. betavasculorum $(P b)$ and $P$. carotovorum $(P c)$ which has been further divided into three subspecies; $P$. carotovorum subsp. carotovorum (Pcc), P. carotovorum subsp. oderiferum (Pco) and P. carotovorum subsp. brasiliensis $(P c b)$. In general, Pectobacterium spp. are broad host range pathogens infecting crop and ornamental plants including both monocots and dicots spanning over 35\% angiosperms plant species (Ma et al., 2007). However, $P$. betavasculorum and $P$. atrosepticum are an exception as they appear to have a much narrower host range almost exclusively restricted to sugar beet and potato, respectively.

Geographically, Pectobacterium carotovorum subspecies carotovorum and $P$. atrosepticum are important pathogens of potatoes in temperate regions causing tuber soft rot and blackleg diseases. Pectobacterium carotovorum subsp. carotovorum has been previously isolated from potatoes in South Africa but to date no P. atrosepticum has been identified (Serfontein et al., 1991). Pectobacterium carotovorum subsp. brasiliensis was first reported in Brazil in 2004 and later shown to occur in other regions including Israel and USA (Duarte et al., 2004; Ma et al., 2007). A recent survey of pectolytic bacteria infecting potatoes in 
South Africa also identified presence of Pectobacterium carotovorum subsp. brasiliensis (van der Merwe et al., 2010). Pectobacterium wasabiae, originally isolated from horse radish, has been shown to cause diseases in potatoes and ornamental plants in the USA, New Zealand and Iran (Goto and Matsumoto, 1987; Kim et al., 2009; Pitman et al., 2008; 2010; BaghaeeRavari et al., 2011). However, there has been no previous report of $P$. wasabiae in South Africa.

Pectobacterium species that have been shown to occur in potatoes are $P$. atrosepticum, $P$. wasabiae, $P$. carotovorum subspp. carotovorum and brasiliensis causing blackleg, tuber soft rot, stem wilt and stem rot symptoms (Kim et al., 2009; Pitman et al., 2010; van der Merwe et al., 2010; Baghaee-Ravari et al., 2011). Because there is an overlap in symptoms caused by the SRE, it is nearly impossible to identify the causal agent by looking at symptoms. Hence biochemical and genetic methods of identification are required in order to accurately identify the relevant causal agent. The SRE are typically seed potato borne pathogens and transmission is mainly through the movement of latently infected seed tubers (Pérombelon and Kelman 1980; Tsror et al., 2009). This movement often leads to the spread of pathogens occurring over long distances as well as across national borders (Toth et al., 2011). As a result, new atypical and highly pathogenic Pectobacterium spp. strains have been identified and these are associated with new potato disease outbreaks in different countries (Pitman et al., 2008; van der Merwe et al., 2010; Baghaee-Baghari et al., 2011). For this reason, it is important that new potentially invasive species are identified and characterised to minimise the impact of the SRE on potato productivity worldwide.

In this study, new, highly invasive, atypical strains of Pectobacterium isolated from potato fields causing potato soft rot and blackleg were identified as $P$. wasabiae. This is the first report of $P$. wasabiae occurring in potatoes in South Africa. 


\section{Materials and Methods}

\section{Bacteria strains}

During the 2010/2011 potato growing seasons, samples of plants showing blackleg and potato tubers showing soft rot symptoms as well as those without symptoms were obtained from the Free State potato growing region of South Africa. Bacterial strains were isolated from tubers and plants with or without symptoms. Type strains were obtained from the International Collection of Belgium and that of the University of Pretoria. The type strains were $P$. carotovorum subsp. carotovorum (LMG $2404^{\mathrm{T}}$ ), $P$. atrosepticum $\left(\mathrm{LMG} 2386^{\mathrm{T}}\right.$ ), $P$. carotovorum subsp. brasiliensis 371 (LMG 21373), P. carotovorum. subsp. brasiliensis 212 (LMG 21371), P. betavasculorum (LMG 2466 ${ }^{\mathrm{T}}$ ), P. wasabiae (LMG $8444^{\mathrm{T}}$ ) and Dickeya spp. (LMG $\left.2804^{\mathrm{T}}\right)$.

\section{Media and culture conditions}

Bacteria were isolated from samples showing blackleg and soft rot symptoms by blending plant tissue in $\mathrm{dH}_{2} \mathrm{O}$ using a blender. Crushed infected material was homogenised and serial dilutions were made with $10 \mathrm{mM} \mathrm{MgSO}_{4}$. Serial dilutions were plated on Crystal Violet Pectate (CVP) (Hyman et al., 2001) and plates were routinely incubated at 27 and $37{ }^{\circ} \mathrm{C}$ for $24-48 \mathrm{~h}$. Single pit forming colonies were transferred to nutrient agar in order to obtain pure colonies for further characterisation. A total of 92 isolates were obtained. All isolates were stored at $-80{ }^{\circ} \mathrm{C}$ in $10 \%$ glycerol. 


\section{Phenotypic and Biochemical characterisation of isolates}

The carbon utilisation profiles of isolated pectolytic bacterial strains were determined using Biolog GN microplates (Biolog, Haywood, CA, USA). Single colonies of each isolate were transferred from nutrient agar and streaked onto tryptone soy agar (TSA) at $27{ }^{\circ} \mathrm{C}$ for $48 \mathrm{~h}$. Colonies were then scraped off TSA plates and suspended in sterile saline as described by Toth et al. (1999) and the OD adjusted to 0.3. Thereafter, $150 \mu 1$ bacterial suspension of each isolate was dispensed into each of the 96 well-microplate and incubated at $27{ }^{\circ} \mathrm{C}$ for $16 \mathrm{~h}$. Readings were taken at OD (595nm) with a Multiscan GO (Thermoscientific) microtitre plate reader.

\section{Molecular identification}

DNA was extracted using standard DNA extraction methods (Sambrook and Russell, 2001). All the primers used in this study were obtained from Inqaba Biotechnologies (South Africa) and are listed in Table 1. All cavity-forming isolates were PCR-amplified with primers Y1 and Y2 specific for the pel gene. Specific PCR assays were carried out to differentiate Pcb and $\mathrm{Pa}$ isolates using primers Eca1f and Eca2r and Br1f and L1r, respectively (Table 1). All strains that were unable to grow at $37{ }^{\circ} \mathrm{C}$ were PCR amplified with $P$. atrosepticum specific primers while all strains that were able to grow at $37{ }^{\circ} \mathrm{C}$ were amplified with $P$. carotovorum subsp. brasiliensis specific primers (Duarte et al. 2004). The PCR reaction was performed in a total volume of $25 \mu \mathrm{l}$ consisting of 10x DreamTaq buffer (supplemented with $20 \mathrm{mM}$ $\mathrm{MgCl}_{2}$ ), $2.5 \mathrm{mM}$ dNTPs, $10 \mu \mathrm{M}$ each forward and reverse primer (Br1f and L1r), $0.5 \mathrm{U}$ DreamTaq Polymerase (Fermentas) and $100 \mathrm{ng}$ DNA template. PCR amplification was conducted using a Biometra Thermocycler (Germany) with the following thermal regime; initial denaturing for $2 \mathrm{~min}$ at $95{ }^{\circ} \mathrm{C}, 30$ cycles of denaturing at $94{ }^{\circ} \mathrm{C}$ for $30 \mathrm{sec}$, followed by 
annealing at $62{ }^{\circ} \mathrm{C}$ for $45 \mathrm{sec}$, and elongation at $72{ }^{\circ} \mathrm{C}$ for $90 \mathrm{sec}$ followed by a final extension step at $72{ }^{\circ} \mathrm{C}$ for $7 \mathrm{~min}$. Amplification products were stained with GelRed (Biotium, Hayward, California, USA) and separated by electrophoresis on $1 \%(\mathrm{w} / \mathrm{v})$ agarose gel (Lonza, USA).

The 16S-23S intergenic transcribed spacer (ITS) region was PCR amplified using primers L1 and G1 according to Toth et al., 2001. Each reaction was made to a final volume of $50 \mu \mathrm{l}$ using $50 \mathrm{ng}$ DNA template, $2 \mathrm{U}$ Taq Polymerase (Fermentas), 5.0 $\mu$ l of 10x Taq DNA polymerase reaction buffer (Fermentas), $200 \mu \mathrm{M}$ dNTPs and $0.4 \mu \mathrm{M}$ each forward and reverse primer. The amplification conditions were as follows: denaturation at $94{ }^{\circ} \mathrm{C}$ for 5 min, 35 cycles of denaturation at $94{ }^{\circ} \mathrm{C}$ for $1 \mathrm{~min}$, annealing at $55{ }^{\circ} \mathrm{C}$ for $2 \mathrm{~min}$ and extension at $72{ }^{\circ} \mathrm{C}$ for $2 \mathrm{~min}$. PCR products were electrophesed on a $2 \%(\mathrm{w} / \mathrm{v})$ agarose gel.

\section{Sequencing and sequence analysis}

Primers designed to anneal to conserved regions of the malate dehydrogenase $(m d h)$ and glyceraldehyde-3- phosphate dehydrogenase A (gapA) genes were used for PCR amplification of representative strains. The PCR reactions were performed in a total volume of $50 \mu \mathrm{l}$ using $50 \mathrm{ng}$ DNA template, and 2U Taq Polymerase (Fermentas), $5 \mu \mathrm{l}$ of 10x Taq DNA polymerase reaction buffer (Fermentas), $200 \mu \mathrm{M}$ dNTPs and $0.4 \mu \mathrm{M}$ forward and reverse primers each. The PCR reaction was carried out using Biometra, Analytik Jena thermocycler and reaction conditions were as follows: initial denaturation at $94{ }^{\circ} \mathrm{C}$ for $30 \mathrm{sec}$, 35 cycles of denaturation at $94{ }^{\circ} \mathrm{C}$ for $30 \mathrm{sec}$ annealing at $52{ }^{\circ} \mathrm{C}$ for $30 \mathrm{sec}$ and extension at $72{ }^{\circ} \mathrm{C}$ for $60 \mathrm{sec}$ followed by a terminal extension at $72^{\circ} \mathrm{C}$ for $7 \mathrm{~min}$. All amplified PCR products were stained with Gelred ${ }^{\circledR}$ and confirmed by gel electrophoresis on a $1 \%$ agarose gel. 
All PCR amplicons were purified using Wizard ${ }^{\circledR}$ SV Cleanup System (Promega) and sequenced using $\mathrm{ABI} 3500 \mathrm{xl}$ at the University of Pretoria. DNA sequences were manually edited using Bioedit v7.1.3 and aligned using ClustalW with a gap opening penalty of 10 and gap extension penalty of 0.2 for multiple alignments. For comparison, reference sequences of Pectobacterium, Dickeya and outgroup sequences of Yersinia species were obtained from the GenBank database (Ma et. al., 2007). Phylogenetic analysis of aligned gene sequences was conducted using MEGAv5.0 (Tamura et al., 2011) and PAUP* through maximum likelihood and maximum parsimony methods on individual and combined data sets. A 1000 parsimony bootstrap replicates were used to assess the stability of clusters. Sequences obtained in this study were deposited in GenBank and accession numbers were obtained (JX312158 JX312169).

\section{Pathogenicity assays}

The virulence of South African isolates was analysed alongside that of the type strains on both potato tubers and stems of potato (Solanum tuberosum) cultivar Mondial. Potato tubers were surface sterilised in $10 \%(\mathrm{v} / \mathrm{v})$ sodium hypochlorite and Tween 20 for $20 \mathrm{~min}$. Each tuber was then inoculated with $10 \mu \mathrm{l}\left(1 \times 10^{8} \mathrm{cfu}^{\mathrm{m} l^{-1}}\right)$ overnight culture of each strain grown on Luria Bertani broth for $16 \mathrm{~h}$ at $27{ }^{\circ} \mathrm{C}$ for $P$. wasabiae and P. atrosepticum strains and 37 ${ }^{\circ} \mathrm{C}$ for P. carotovorum subsp. carotovorum and P. carotovorum subsp. brasiliensis strains. Using a sterile pipette tip, holes of a uniform depths were stabbed into potato tubers and $10 \mu \mathrm{l}$ inoculum injected. The inoculum was sealed with Vaseline (Petroleum Jelly) and inoculated tubers were incubated at $25{ }^{\circ} \mathrm{C}$. After $72 \mathrm{~h}$, rotting tissue was scraped from the tubers and weighed to determine the extent of tissue maceration. This experiment was performed three 
independent times using a total of seven tubers per trial. Statistical significance was analysed by T-Test or ANOVA (JMP v5).

To evaluate stem rot and blackleg, five week old potato plants Solanum tuberosum (cv Mondial) were inoculated with $10 \mu \mathrm{l}\left(1 \times 10^{8} \mathrm{cfu}^{\mathrm{m} l^{-1}}\right)$ of overnight bacterial cultures (using the same strains used for tuber soft rot assays) suspended in $10 \mathrm{mM} \mathrm{MgSO}$. Plants were inoculated by piercing the stem with a micropipette approximately $10 \mathrm{~cm}$ above the soil level. Inoculation sites were immediately wrapped with parafilm to prevent desiccation. Plants were incubated in a glasshouse at $25{ }^{\circ} \mathrm{C}$ at high humidity and blackleg lesions were evaluated daily for 21 days post inoculation (dpi). Three plants each consisting of 2-3 stems were inoculated per strain.

\section{Hypersensitive response}

Hypersensitive response of the type strains of $P$. carotovorum subsp. brasiliensis (positive control), P. atrosepticum (negative control), P. wasabiae and selected South African strains was evaluated on tobacco leaves (Nicotiana benthamiana and N. tabaccum [Xanthi]). A negative control of $10 \mathrm{mM} \mathrm{MgSO}_{4}$ was included for each experiment. Overnight cultures $(1 \mathrm{x}$

$10^{8} \mathrm{cfu}^{-1} \mathrm{ml}^{-1}$ ) were washed twice and re-suspended in $10 \mathrm{mM} \mathrm{MgSO}$. Tobacco leaves were then infiltrated with bacterial suspension and inoculated plants were maintained at room temperature for $24-48 \mathrm{~h}$. Hypersensitive response was recorded at 48 hours post inoculation (hpi). Three leaves of two plants each N. tabaccum and N. benthamiana were infiltrated and two independent biological replicates were performed.

\section{RESULTS}




\section{Identification of pectolytic bacteria}

Potato stems and tubers with or without typical symptoms of blackleg and tuber soft rot were obtained from the Free State potato growing region of South Africa. Isolation of pectolytic bacterial strains was carried out on CVP and in total, 92 isolates of cavity forming bacteria were obtained. Of the samples tested, pectolytic bacteria were isolated from 8 sample batches consisting of either rotting seed potato tubers from generations $4-6(\mathrm{G} 4-\mathrm{G} 6)$ of production or stems with blackleg symptoms. On average, approximately 12 pectolytic bacteria were isolated per sample. All isolates were further identified as pectolytic bacteria by PCR amplification of the pel gene using Y1 and Y2 primers. The expected amplicon of $434 \mathrm{bp}$ was obtained for all isolates. Nearly half $(52 \%)$ of the isolates were able to grow at both $27{ }^{\circ} \mathrm{C}$ and $37{ }^{\circ} \mathrm{C}$ while another half $(48 \%)$ were not able to grow at $37{ }^{\circ} \mathrm{C}$. The type strains of $P$. atrosepticum and $P$. wasabiae were also not able to grow at this elevated temperature. Carbon utilisation profiles of these isolates could not be used to conclusively differentiate them from those of $P$. carotovorum subsp. carotovorum (results not shown). Hence, further characterisation of these isolates using other techniques was deemed necessary.

\section{Molecular typing of Pectobacterium spp. isolates}

The newly isolated Pectobacterium spp. were further characterised using species and subspecies specific primers (Eca1f and Eca2r and Br1 and L1R) for P. atrosepticum and $P$. carotovorum subsp. brasiliensis respectively. The expected amplicon of 322 bp was obtained from the type strains of $P$. carotovorum subsp. brasiliensis as well as all 48 isolates that were able to grow at both $27{ }^{\circ} \mathrm{C}$ and $37{ }^{\circ} \mathrm{C}$. Thus this group of isolates could positively be identified as $P$. carotovorum subsp. brasiliensis using species specific primers. To characterise the remaining group of isolates, these were subjected to PCR amplification using 
P. atrosepticum specific primers. None of these strains could amplify the expected amplicon of $690 \mathrm{bp}$ which was observed only for the type strain of P. atrosepticum (results not shown). Given that carbon profiles of these isolates were similar to those of P. carotovorum subsp. carotovorum but they could not grow at $37^{\circ} \mathrm{C}$ and failed to amplify with $P$. atrosepticum specific primers, these isolates were labelled as atypical. Five of these atypical isolates, G63 and G67 representing isolates from stem tissue and GT6, GT13 and GT9 representing isolates from infected seed tubers were selected for further characterisation.

The ITS regions of the five selected atypical strains from South Africa including the type strains for $P$. carotovorum carotovorum, $P$. betavasculorum, $P$. atrosepticum, $P$. carotovorum subsp. brasiliensis, P. wasabiae and D. dadantii were PCR-amplified using general primers L1 and G1. The ITS-PCR profiles of the type strains of P. atrosepticum and D. dadantii were clearly different from all other strains (results not included). However, there was no clear difference between ITS-PCR profiles of atypical South African strains and those of $P$. carotovorum subsp. carotovorum, $P$. betavasculorum and $P$. carotovorum subsp. brasiliensis type strains. To enable us to distinguish atypical South African strains from the type strains of $P$. carotovorum subsp. carotovorum, $P$. betavasculorum and $P$. carotovorum subsp. brasiliensis strains, ITS-PCR products were digested with two different restriction digest enzymes (CfoI or RsaI) and the resulting PCR-RFLP profiles were compared. The PCR-RFLP banding profiles generated with RsaI could clearly differentiate between the five selected atypical strains (Figure 1A lanes $1-5$ ) and the type strains of $D$. dadantii, $P$. atrosepticum, P. betavasculorum, P. carotovorum subsp. carotovorum and P. carotovorum subsp. brasiliences strain 357 . However, the profiles of the atypical strains generated using RsaI could not be used to conclusively distinguish between our strains and those of $P$. wasabiae type strain and P. carotovorum subsp. brasiliensis strain 358 (Figure 1A). Thus, to further identify these isolates, ITS-PCR-RFLP profiles were generated using a second 
restriction digest enzyme, CfoI. Using this enzyme, the five atypical strains could clearly be distinguished from other Pectobacteriums spp. type strains (Figure 1B 1-2 and 4-6) and were identified as being most similar to those of $P$. wasabiae type strain (Figure 1B).

\section{Phylogenetic analysis of atypical South African strains}

The use of partial housekeeping genes such as the malate dehydrogenase $(m d h)$ and gluteraldehyde-3-phosphate A (gapA) genes has been widely used for phylogenetic characterisation of the SRE (Ma et al. 2007; Pitman et al., 2010). Thus, these genes were amplified from representative South African strains identified as $P$. carotovorum subsp. brasiliensis and $P$. wasabiae together with representative type strains, sequenced and aligned using clustalW. Maximum likelihood trees were constructed using individual genes and concatenated sequences of the two genes, $m d h$ and gapA (Figure 2). In the concatenated phylogentic tree in Figure 2, all of the isolates that were identified by species specific primers as $P$. carotovorum subsp. brasiliensis clustered closely with those of $P$. carotovorum subsp. brasiliensis isolated elsewhere in clade I. The clade was supported with a high bootstrap value of $97 \%$. On the other hand, all the South African atypical strains identified as $P$. wasabiae using ITS-PCR-RFLP clustered closely together with those of $P$. wasabiae type and reference strains in clade IV. The bootstrap support value of this clade was $94 \%$ indicating high levels of confidence for the clade. The phylogenetic tree constructed provided evidence supporting identification of these isolates as $P$. wasabiae. This further substantiated PCR-RFLP identification of these isolates as $P$. wasabiae. This is the first report of $P$. wasabiae in potatoes in South Africa. Previous reports of $P$. wasabiae strains infecting potatoes were in the USA, New Zealand and Iran (Kim et al., 2009; Pitman et al., 2008; Baghaee-Ravari et al., 2011). 


\section{Virulence and hypersensitive response}

The relative virulence of South African strains identified as $P$. wasabiae was compared to those of the type strains of $P$. atrosepticum, $P$. carotovorum subsp. carotovorum, $P$. wasabiae and P. carotovorum subsp. brasiliensis by inoculation on potato tubers and stems (Solanum tuberosum cv Mondial). Tuber maceration was quantified by scooping and weighing rotting tissue. As expected, there was no rotting observed where potato tubers were inoculated with the negative control, $\mathrm{MgSO}_{4}$. The highest average tissue maceration was observed for $P$. carotovorum subsp. carotovorum and $P$. carotovorum subsp. brasiliensis type strains including one South African P. wasabiae strain SA G620 (Figure 3). The other five South African $P$. wasabiae strains showed variable maceration ability which was significantly higher than the type strains of $P$. wasabiae and $P$. atrosepticum. In stem assays, mock inoculated stems remained without symptoms for the entire duration of the experiment (Figure 4A). Typical blackleg symptoms appeared on inoculated stems within 3 dpi in the form of black lesions extending upwards and downwards from the point of inoculation (Figure 4B and C). In some cases, severe wilting and complete collapse of the plant was observed (Figure 4C).

The type III secretion system (T3SS) is an important pathogenicity factor of many Gram negative bacteria. Effector proteins of the T3SS when injected into non host plants elicit a hypersensitive response. Atypical P. wasabiae and Pseudomonas syringae strains lacking a functional T3SS or with a highly evolved T3SS have been isolated previously (Kim et al., 2009; Clarke et al., 2010; Pitman et al., 2010). Typically, such strains lack the ability to elicit HR when inoculated into tobacco plants. Thus, the ability of five $P$. wasabiae strains isolated from South Africa to elicit hypersensitive response on tobacco plants was 
investigated. Six weeks old N. tabaccum (cv Xanthi) and N. benthamiana plants were infiltrated with $1 \times 10^{8} \mathrm{cfu}_{\mathrm{ml}}^{-1}$ cells of the type strains of $P$. wasabiae and $P$. carotovorum subsp. brasiliensis (used as a positive control), P. atrosepticum and $\mathrm{MgSO}_{4}$ (included as negative controls) together with the South African P. wasabiae strains G63, G67, GT6, GT13 and GT19. At 24 hpi, typical tissue collapse indicative of HR, could be detected in areas infiltrated with $P$. carotovorum subsp. brasiliensis but as expected, no signs of tissue collapse were visible in areas infiltrated with $\mathrm{MgSO}_{4}$ or $P$. atrosepticum (Figure 5). Furthermore, no HR was observed in areas infiltrated with $P$. wasabiae type strain nor any of the South African P. wasabiae strains.

\section{DISCUSSION}

In this study, potato tubers and stems showing symptoms of blackleg and potato soft rot were collected and the causal agent identified. Using a combination of physiological, biochemical and molecular diagnostic techniques, we were able to show that $52 \%$ of the strains were Pectobacterium carotovorum subsp. brasiliensis which has previously been reported as the main cause of potato blackleg and tuber soft in potato fields in South Africa (van der Merwe et al., 2010). However, we identified a second group of isolates whose identity could not be resolved by carbon utilisation profiles generated using biolog assays. These isolates were initially described as atypical $P$. carotorovorum subsp. carotovorum because, unlike typical P. carotovorum subsp. carotovorum strains, they could not grow at $37{ }^{\circ} \mathrm{C}$. Several studies have shown that physiological and biochemical methods on their own are often unable to accurately discriminate between SRE species, particularly those closely related to $P$. carotovorum subsp. carotovorum (Toth et al., 1999; Pitman et al., 2008). It has also been shown that although biolog has been used widely for identification of other bacteria, it cannot 
clearly distinguish between related members of the Pectobacterium spp. (Toth et al., 1999). Considering that $P$. atrosepticum isolates like our isolates, cannot grow at the elevated temperature of $37^{\circ} \mathrm{C}$, PCR amplification of these isolates using species specific primers for P. atrosepticum was attempted. However, none of these isolates amplified with these primers. Thus, other molecular diagnostic tools were selected for use to further characterise atypical South African isolates.

Molecular diagnostic tools such as species specific primers, ITS-PCR and PCR-RFLP have been successfully used to resolve identities of the SRE that could not be resolved using biochemical and physiological characteristics (Toth et al., 2001). Thus ITS-PCR and PCRRFLP were used to further resolve the identities of these strains. For this purpose, five of these isolates were selected. The profiles generated using the restriction enzyme RsaI could discriminate between our isolates and those of Dickeya spp., P. atrosepticum, $P$. carotovorum subsp. carotovorum and one $P$. carotovorum brasiliensis strain. However, we could not discriminate between our isolates and another strain of $P$. carotovorum subsp. brasiliensis or $P$. wasabiae. The use of CfoI was better able to identify our isolates as $P$. wasabiae. This was further confirmed using maximum likelihood phylogenetic analysis which showed that the atypical South African strains clustered closely to those of $P$. wasabiae strains isolated from other geographic locations. Strains that were positively identified as P. carotovorum subsp. brasiliensis using species specific primers clustered closely within the P. carorotovorum subsp. brasiliensis clade. Both $P$. wasabiae and $P$. carorotovorum subsp. brasiliensis were often isolated together as a mixed population from a single batch of samples, and from our observations, there was no clear 'preference' for stem or tuber by either strain.

Pectobacterium wasabiae was originally isolated from horse radish. However, there have been several reports of its occurrence in potatoes (Ma et al., 2007; Pitman et al., 2008; Kim et al., 2009; Baghaee-Ravari et al., 2011). Interestingly, Ma et al. (2007) previously 
identified several $P$. wasabiae strains lacking a type III secretion system (T3SS). Pitman et al (2010) also isolated $P$. wasabiae strains which elicited a mild form of hypersensitive response in Nicotiana tabaccum (cv Xanthi). They showed that these strains also lacked key effectorencoding genes such as $d s p E$ and $h r p N$. Thus we sought to determine whether the South African strains could elicit HR. None of the South African P. wasabiae isolates were able to elicit hypersensitive response in $N$. benthamiana or $N$. tabaccum. The same observation was made for $P$. wasabiae and $P$. atrosepticum type strains. However, the type strain and all South African P. carotovorum subsp. brasiliensis isolates were able to elicit a form of tissue collapse within 24 hpi in both $N$. benthamiana and $N$. tabaccum (results not included). These results are in keeping with earlier observations by Glasner et al. (2008) who previously reported that both $P$. carotovorum subspp. carotovorum and brasiliensis were able to elicit HR while P. atrosepticum (SCRI 1043) could not. Similar to Ma et al. (2007), our study indicated a lack of HR elicitation by all five South African $P$. wasabiae isolates that were selected for HR assays, suggesting a possible lack of some key genes of T3SS if not the entire cluster. A PCR screening of at least two genes ( $h r p N$ and $d s p E)$ with primers designed based on conserved gene regions could not detect presence of these genes in the South African $P$. wasabiae isolates (results not included). It is important to note that the absence of PCR amplicons cannot be used as conclusive evidence that these isolates lack a T3SS. Further work is required to verify this. Although $P$. wasabiae strains isolated in South Africa did not elicit HR in Nicotiana plants, they were highly virulent with one strain causing rot equivalent to that of $P$. carotovorum subp. carotovorum and $P$. carotovorum subsp. brasiliensis, both of which are often reported as being the more aggressive Pectobacterium spp. on potatoes (Marquez-Villavicencio et al., 2011). Furthermore, all the selected strains tested were virulent on potato plant stems causing mild to aggressive forms of blackleg and wilting leading in some cases to complete collapse of the plant. Thus, it appears that there was no 
correlation between the presence or absence of a T3SS in these strains and the ability to cause disease. These observations are consistent with those of other researchers (Ma et al. 2007; Kim et al., 2009; Pitman et al. 2010; Baghaee-ravari et al. 2011).

In conclusion, our study identified $P$. wasabiae strains causing potato blackleg and soft rot symptoms in South African potatoes using a combination of molecular and phylogenetic analyses. These strains, although not able to elicit HR in tobacco plants remained aggressive on potato stems and tubers. Survival and virulence mechanisms used by these T3SS-deficient Pectobacterium spp. in comparison to other Pectobacterium spp. warrants further research. To our knowledge, this is the first report of $P$. wasabiae in South Africa. Occurrence of this pathogen in other potato growing regions will need to be investigated.

\section{Acknowledgements}

\section{This work was funded by the National Research Foundation}




\section{References}

Baghaee -Ravari S., Rahimian, H, Shams-Bakhsh, M., Lopez-Solanilla E., Antunez-Lamaz M., \& Rodriguez-Penzuela, P. (2011) Characterization of Pectobacterium species from Iran using biochemical and molecular methods. European Journal of Plant Pathology $129,413-425$

Charkowski, A., Blanco, C., Condemine, G., Expert, D., Franza, T., Hayes, C. et al. (2012) The role of secretion systems and small molecules in soft-rot Enterobacteriaceae pathogenicity. Annual Review of Phytopathology, ahead of print

Clarke, R. C., Cai, R., Studholme, D. J., Guttman, D. S., \& Vinatzer, B. A. (2010) Pseudomonas syringae strains naturally lacking the classical $P$. syringae hrp/hrc locus are common leaf colonizers equipped with an atypical Type III Secretion System. Molecular Plant Microbe Interaction 23(2), 198-210.

Duarte, V., de Boer, S. H., Ward, T. L., de Oliveora, A. M. R. (2004) Characterisation of atypical Erwinia carotovora subsp. atroseptica causing blackleg of potato in Brazil. Journal of Applied Microbiology 96, 535-545

Gardan, L., Gouy, C., Christen, R., \& Samson, R. (2003) Elevation of three subsp. of Pectobacterium to subsp. level: Pectobacterium atrosepticum sp. nov., Pectobacterium betavasculorum sp. nov. and Pectobacterium wasabiaes p. nov. International Journal of Systematic and Evolutionary Microbiology, 53, 381-391.

Glasner, J. D., Marquez-Villavicencio, M., Kim, H. S., Jahn, C. E., Ma, B., Biehl, B. S., et al. (2008). Niche-specificity and the variable fraction of the Pectobacterium pan genome. Molecular Plant Microbe Interactions, 21, 1549-1560. 
Goto, M. \& Matsumoto, K. (1987) Erwinia carotovora subsp. wasabiae subsp. nov. isolated from diseased rhizomes and fibrous roots of Japanese horseradish. International Journal of Systematic Bacteriology 37, 130-135

Hauben, L., Moore, E. R., Vauterin, L., Steenackers, M., Mergaert, J., Verdonck, L., et al. (1998). Phylogenetic position of phytopathogens within the Enterobacteriaceae. Systematic and Applied Microbiology, 21, 384-397

Hyman, L. J., Sulivan, L., Toth, I. K., \& Pérombelon, M. C. M., (2001), Modified crystal violet pectate medium (CVP) based on a new polypectate source (Slendid) for the detection and isolation of soft rot erwinias. Potato Research 44, 265-270

Kim, H. S., Ma, B., Perna, N. T., \& Charkowski, A. O. (2009). Phylogeny and virulence of naturally occurring type III secretion system-deficient Pectobacterium strains. Applied and Environmental Microbiology, 75, 4539-4549.

Ma, B., Hibbing, M. E., Kim, H. S., Reedy, R. M., Yedidia L., Breuer J. et al. (2007) Host range and molecular phylogenies of the soft rot enterobacterial genera Pectobacterium and Dickeya. Phytopathology 97, 1150-1163

Marquez-Villavicencio, M. D. P., Groves R.L. \& Charkowski A. O., (2011) Soft rot disease severity is affected by potato physiology and Pectobacterium taxa. Plant Disease 95, $232-241$.

Pérombelon, M.C.M. \& Kelman, A. (1980). Ecology of soft rot erwinias. Annual Review of Phytopathology 18, 361-387 
Pitman, A. R., Wright, P.J., Gailbraith, M. D. \& Harrow, S.A. (2008) Biochemical and genetic diversity of pectolytic enterobacteria causing soft rot disease of potatoes in New Zealand. Australasian Plant Pathology 37, 559-568

Pitman, A. R., Harrow, S. A. and Visnovsky, S. B. (2010).Genetic characterisation of Pectobacterium wasabiae causing soft rot disease of potato in New Zealand. European Journal of Plant Pathology 126, 423-435

Sambrook, J. \& Rusell, D. (2001) Molecular Cloning: A Laboratory Manual 3rd Ed Cold Spring Harbor Lab. Press, Plainview, New York

Samson, R., Legendre, J.B., Christen, R., Fishcer-Le Saux, M., Achouak, W. and Gardan L., (2005), Transfer of Pectobacterium chrysanthemi (Burkholder et al., 1953) Brenner et al. 1973 and chrysanthemi comb. Nov. and Dickeya paradisiaca comb.nov.and delineation of four novel species, Dickeya dadantii sp. nov., Dickeya dianthicola sp. nov. Dickeya dieffenbachiae sp. nov.and Dickeya zeae sp. nov. International Systematics Evolutionary Microbiology, 55,1415-1427

Serfontein S., Logan, C., Swanepoel, A. E., Boelem, B.H., \& Theron, D.J., (1991) A potato wilt disease in South Africa caused by Erwinia carotovora subspecies carotovora and E. chrysanthemi. Plant Pathology 40, 382-386

Tamura K., Peterson D., Peterson N., Stecher G., Masatoshi N. \& Kumar S. (2011) MEGA5: Molecular Evolutionary Genetics Analysis Using Maximum Likelihood, Evolutionary Distance, and Maximum Parsimony Methods Molecular Biology and Evolution, 28, 2731-2739.

Toth I. K., Bertheau Y., Hyman L. J., Laplaze L., Lopez M. M., J. \& McNicol, F. et al., (1999) Evaluation of phenotypic and molecular typing techniques for determining 
diversity in Erwinia carotovora subspp. atroseptica. Journal of Applied Microbiology, $87,770-781$

Toth, I. K., Newton, J. A., Hyman, L., ( 2001), Rapid identification and differentiation of the soft rot erwinias by $16 \mathrm{~S}-23 \mathrm{~S}$ intergenic transcribed spacer PCR and restriction enzyme fragment polymorphism analyses. Applied Environmental Microbiology, 67, 40704076.

Toth, I.K., van der Wolf, J.M., Saddler, G., Lojkowska, E., Helias, E., Pirhonen, M., Tsror (Lahkim), L., Elphistone, J.G., (2011), Dickeya species: an emerging problem for potato production in Europe. Plant Pathology, 60, 385-399

Tsror, L., Erlich, O., Lebuish, S., Hazanovsky, M., Zig, U., Slawiak, M., et al., (2009), Assessment of recent outbreaks of Dickeya sp. (syn. Erwinia chrysanthemi) slow wilt in potato crops in Israel. European Journal of Plant Pathology, 123,311-320.

van der Merwe, J. J., Coutinho, T. A., Korsten, L., van der Waals J. E., (2010) Pectobacterium carotovorum subsp. brasiliensis causing blackleg on potatoes in South Africa. European Journal of Plant Pathology 126, 175-185 


\section{APPENDICES}

\section{Table 1: Primers used in this study}

Figure 1 : ITS-PCR-RFLP profiles of Pectobacterium spp isolates using A. RsaI and B. CfoI restriction restriction enzyme. Ladder is a standard molecular ladder. The South African Pectobacterium strains SA G63; SA G67; SA GT6; SA GT13; SA GT19; were compared to type strains $P$. carotovorum subsp. brasiliensis 357 (Pcb 357); P. wasabiae $\left(P w^{\mathrm{T}}\right)$; P. atrosepticum $\left(\mathrm{Pa}^{\mathrm{T}}\right) ;$ P. betavasculorum $\left(P b^{\mathrm{T}}\right) ; P$. carotovorum subsp. carotovorum

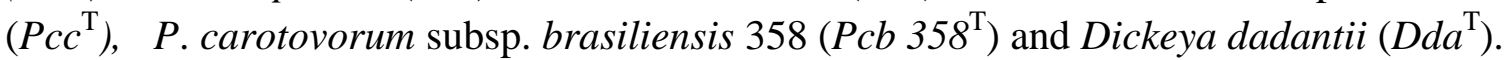

Figure 2: A concatenated maximum likelihood phylogenetic analysis of Pectobacterium spp strains from South Africa based on $\boldsymbol{m d h}$ and gapA partial gene sequences. Numbers represent bootstrap support values greater than 50\% from 1000 replicates using heuristic searches. Yersinia spp. were used as outgroups.

Figure 3: Relative virulence of South African $P$. wasabiae strains. The relative virulence of South Africa $P$. wasabiae strains was evaluated by inoculating the following strains into potato (Solanum tuberosum cv Mondial) tubers: $P$. carotovorum brasiliensis $357 ; \quad P$. wasabiae $\left(P w^{\mathrm{T}}\right) ;$ P. atrosepticum $\left(P a^{\mathrm{T}}\right) ; P$. carotovorum subsp. carotovorum $\left(P c c^{\mathrm{T}}\right)$; South African $P$. wasabiae strains SA GT6, 2; SA GT13, SA GT19, SA G63; SA G67; SA G620 and $\mathrm{MgSO}_{4}$ control.

Figure 4: Potato blackleg symptoms on Solanum tuberosum (cv Mondial) after stem inoculations with A. $\mathrm{MgSO}_{4}$ control; B. SA GT19 and C. SA G620

Figure 5: Hypersensitive response elicitation in tobacco plants. A. Nicotiana tabaccum (cv Xanthi) plants were infiltrated with 1. P. carotovorum subsp. brasiliesis, 2. P. wasabiae and 3. $P$. atrosepticum type strains, $4 . \mathrm{MgSO}_{4}$ and South African. $P$. wasabiae strains 5. SA G63; 6. SA G67; 7. SA GT19; 8. SA G620 B. Nicotiana benthaminana plants were infiltrated with 1. P. carotovorum subsp. brasiliesis, 2. P. wasabiae type strains 3. $\mathrm{MgSO}_{4}$, and South African P. wasabiae strains 4. SA G63; 5. SA G67 and 6. SA GT19 
Table 1: Primers used in this study

\begin{tabular}{|l|l|}
\hline Primer & \multicolumn{1}{|c|}{ Sequence (5' $\mathbf{- 3}$ ') } \\
\hline Eca1f & CGGCATCATAAAAACACG \\
\hline Eca2r & GCACACTTCATCCAGCGA \\
\hline Y1 & TTACCGGACGCGAGCTGTGGCGT \\
\hline Y2 & CAGGAAGATGTCGTTATCGCGAGT \\
\hline Br1f & GCGTGCCGGGTTTATGACCT \\
\hline L1r & CARGGCATCCACCGT \\
\hline L1 & CAAGGCATCCACCGT \\
\hline G1 & GAAGTCGTAACAAGG \\
\hline mdh2 & GCGCGTAAGCCGGGTATGGA \\
\hline mdh4 & CGCGGCAGCCTGGCCCATAG \\
\hline gapA & ATCTTCCTGACCGACGAAACTGC \\
\hline gapA & ACGTCATCTTCGGTGTAACCCAG \\
\hline
\end{tabular}




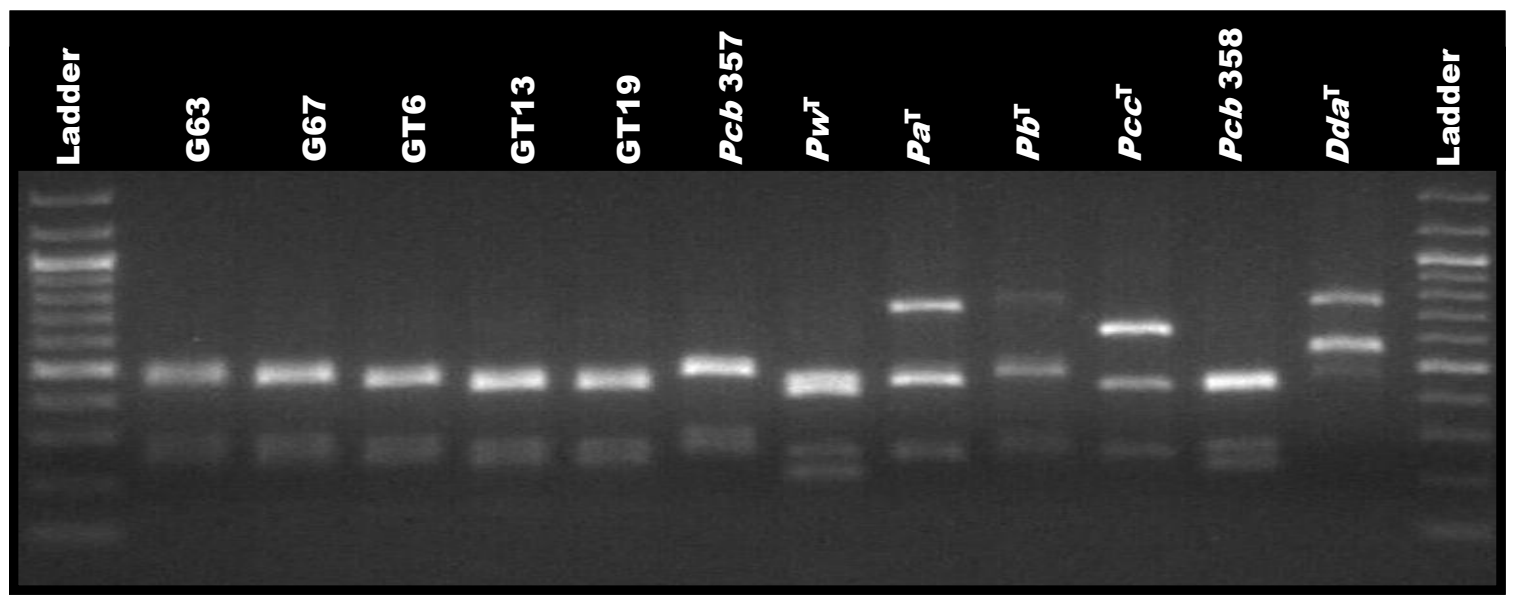

Figure 1A

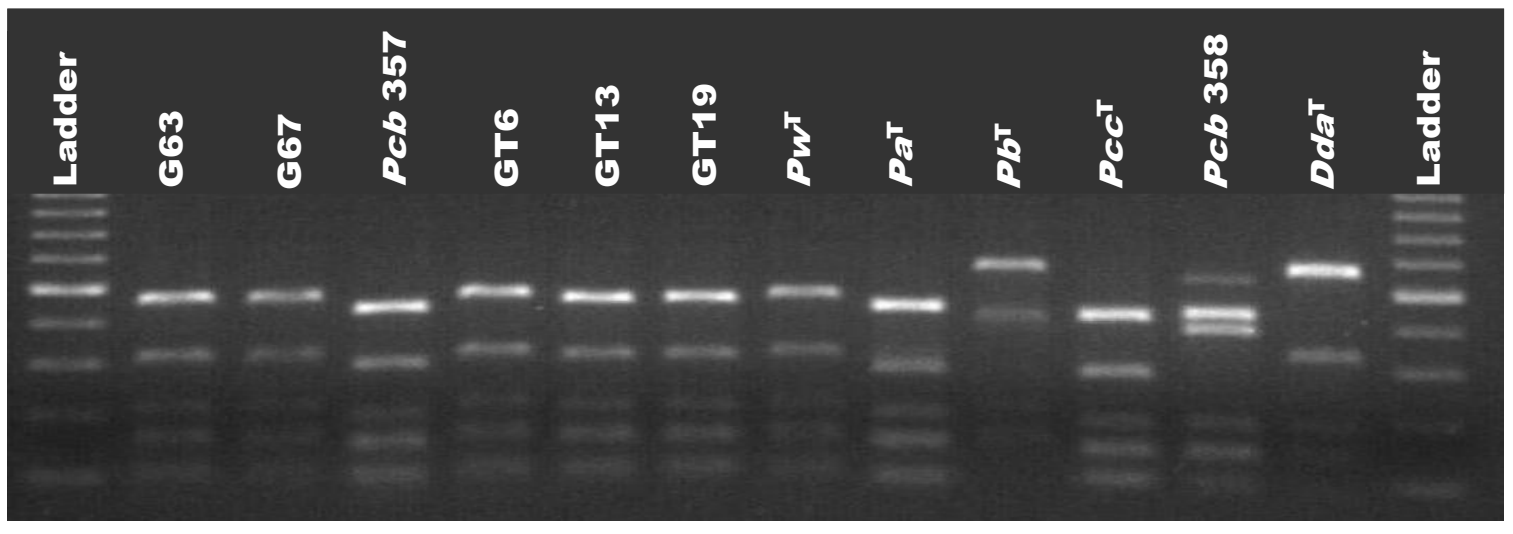

Figure 1B 


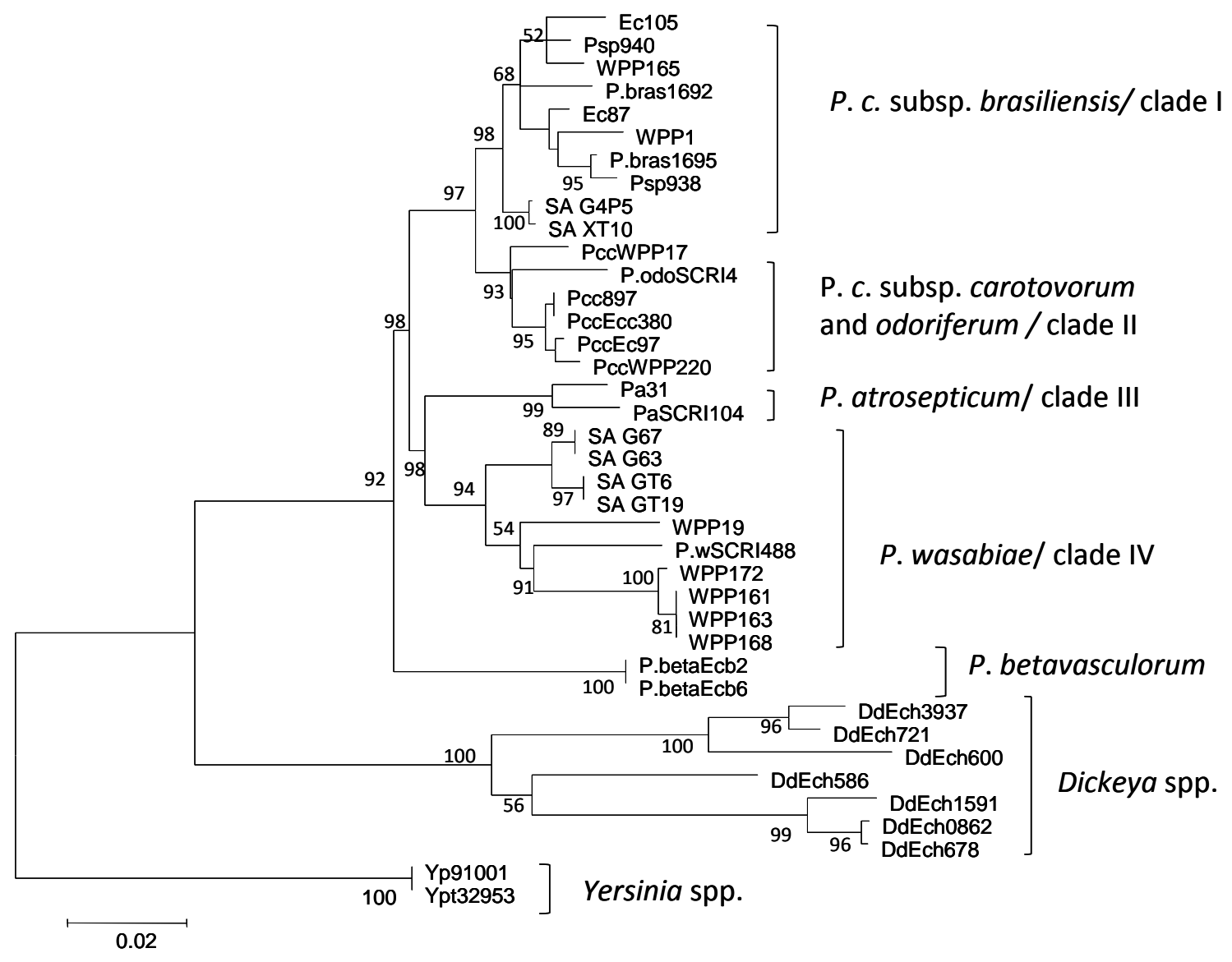

Figure 2 


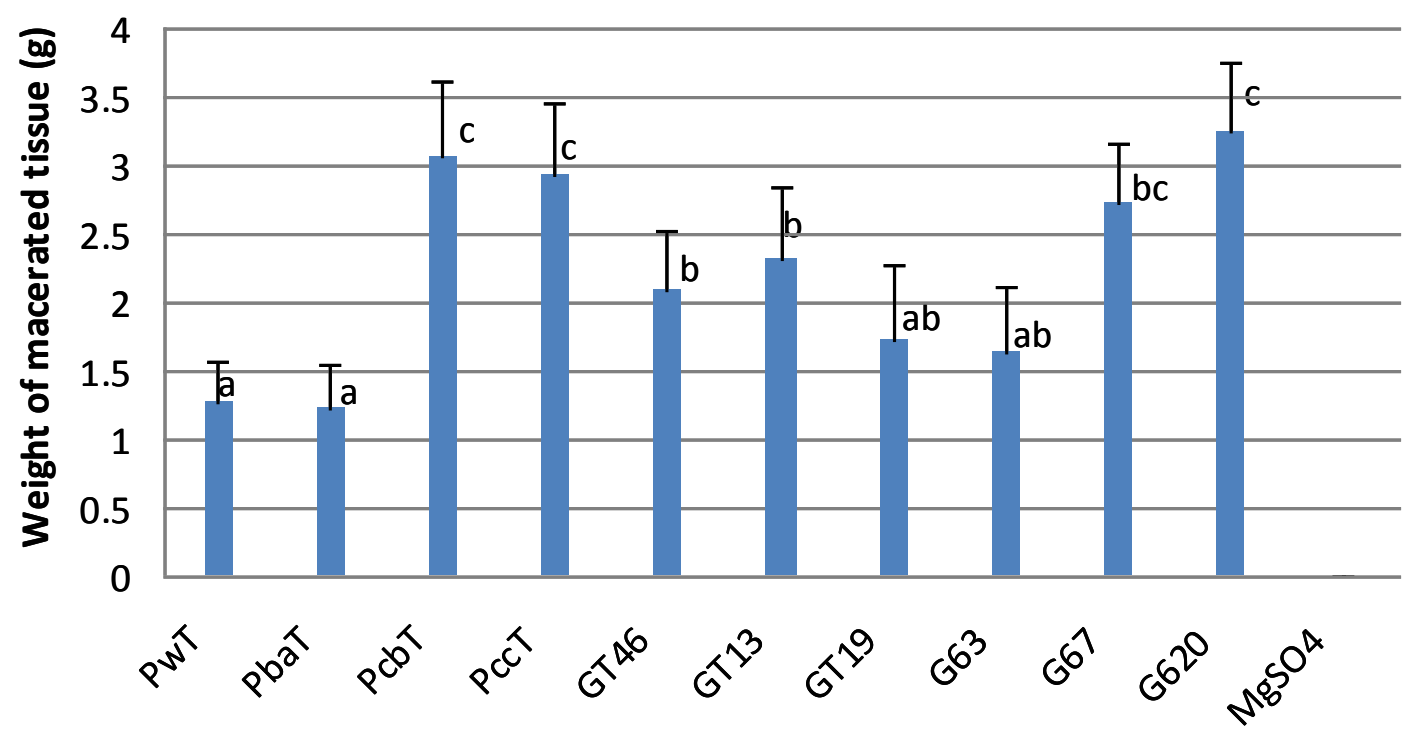

Figure 3 

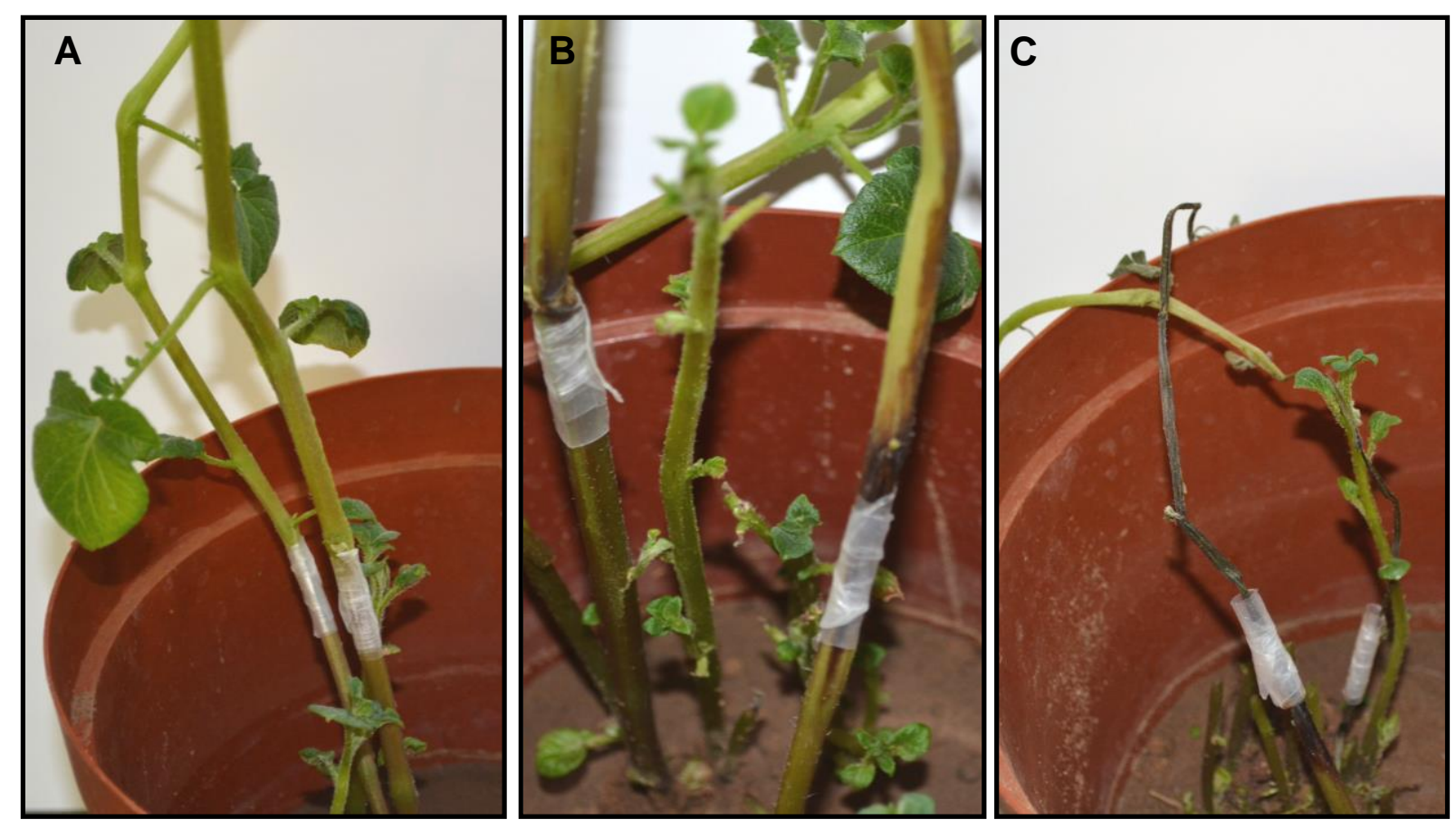

Figure 4 


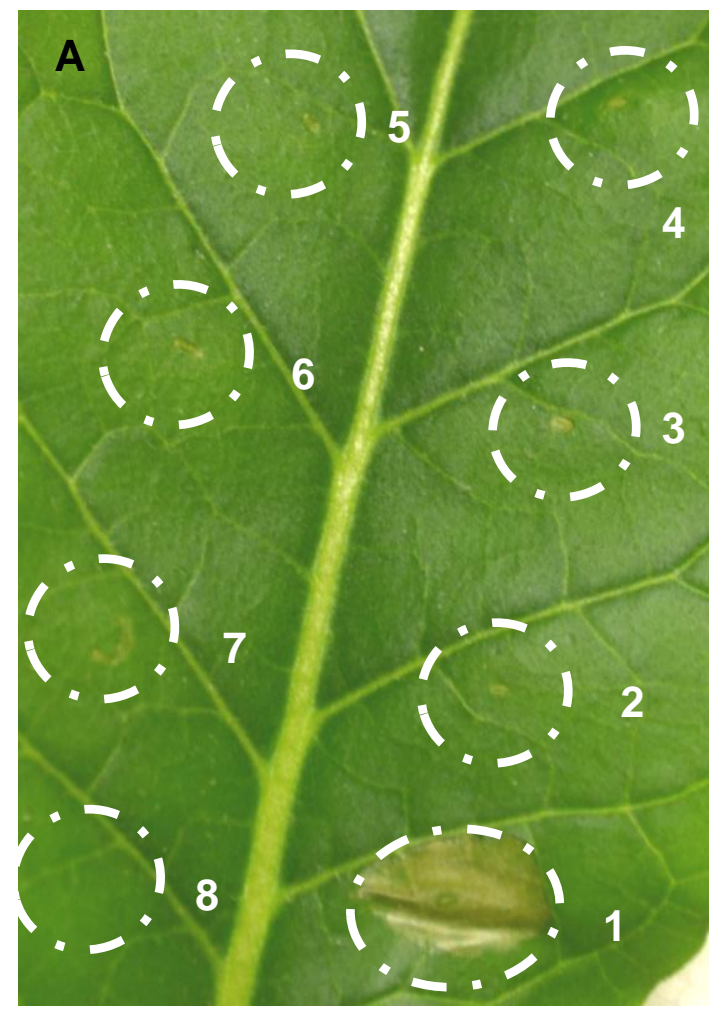

B

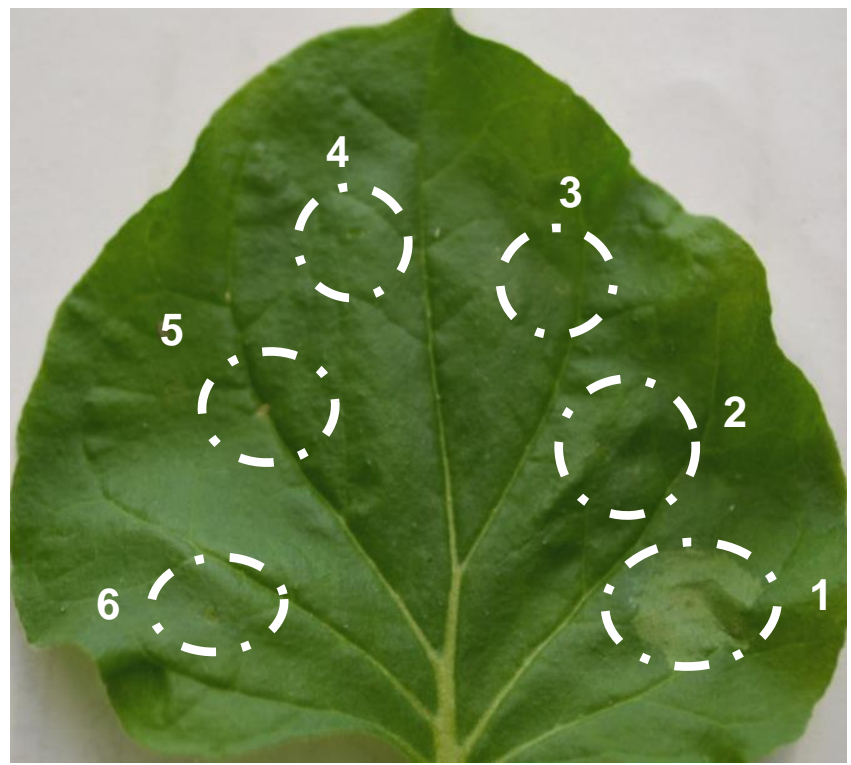

Figure 5 